\title{
Adverse effects of therapeutic hypothermia in a 55-year-old man with cardiac arrest
}

\author{
Andrew D. Moeller MD MASc, Jordan C. Webber MD
}

n Cite as: CMAJ 2017 October 30;189:E1337-40. doi: 10.1503/cmaj.170682

A 55-year-old man, with no history of ischemic heart disease, cardiac risk factors or family history of sudden cardiac death, suddenly collapsed at a local community rink after playing hockey. Bystander cardiopulmonary resuscitation (CPR) was started immediately. Ten minutes later, when paramedics arrived, the patient was in ventricular fibrillation. He was defibrillated successfully and his first electrocardiogram (ECG) showed a narrow junctional escape rhythm at 45 beats/min and inferior ST-segment elevation myocardial infarction (STEMI) (Figure 1). He was transferred to the regional cardiac catheterization centre for percutaneous coronary intervention $(\mathrm{PCl})$ after receiving appropriate medical treatment for acute coronary syndrome.

Coronary angiography showed that the patient had $100 \%$ proximal occlusion of the culprit dominant right coronary artery with an $80 \%$ medium second obtuse marginal lesion (Figure 2). A drugeluting stent was inserted into his proximal right coronary artery. His left ventriculogram showed an ejection fraction of $53 \%$ with basal and mid-inferior hypokinesis. Postcardiac arrest cooling protocol

\section{KEY POINTS}

- Although therapeutic hypothermia is recommended and generally safe, severe and life-threatening complications are possible.

- Prolongation of QTc is a common result of therapeutic hypothermia and typically does not lead to adverse outcomes, but must be monitored closely.

- Current protocols for therapeutic hypothermia in hospitals should be reviewed to take into account new recommendations for a core body temperature range of $33^{\circ} \mathrm{C}$ to $36^{\circ} \mathrm{C}$.

was started in the cardiac catheterization laboratory and the patient was transferred to the coronary care unit. An ECG conducted after $\mathrm{PCl}$ showed sinus rhythm with normal QTc interval and serial changes of revascularized inferior STEMI (Appendix 1, supplemental Figure 1, available at www.cmaj.ca/lookup/suppl/doi:10.1503/ cmaj.170682/-/DC1). While in the coronary care unit, the patient's

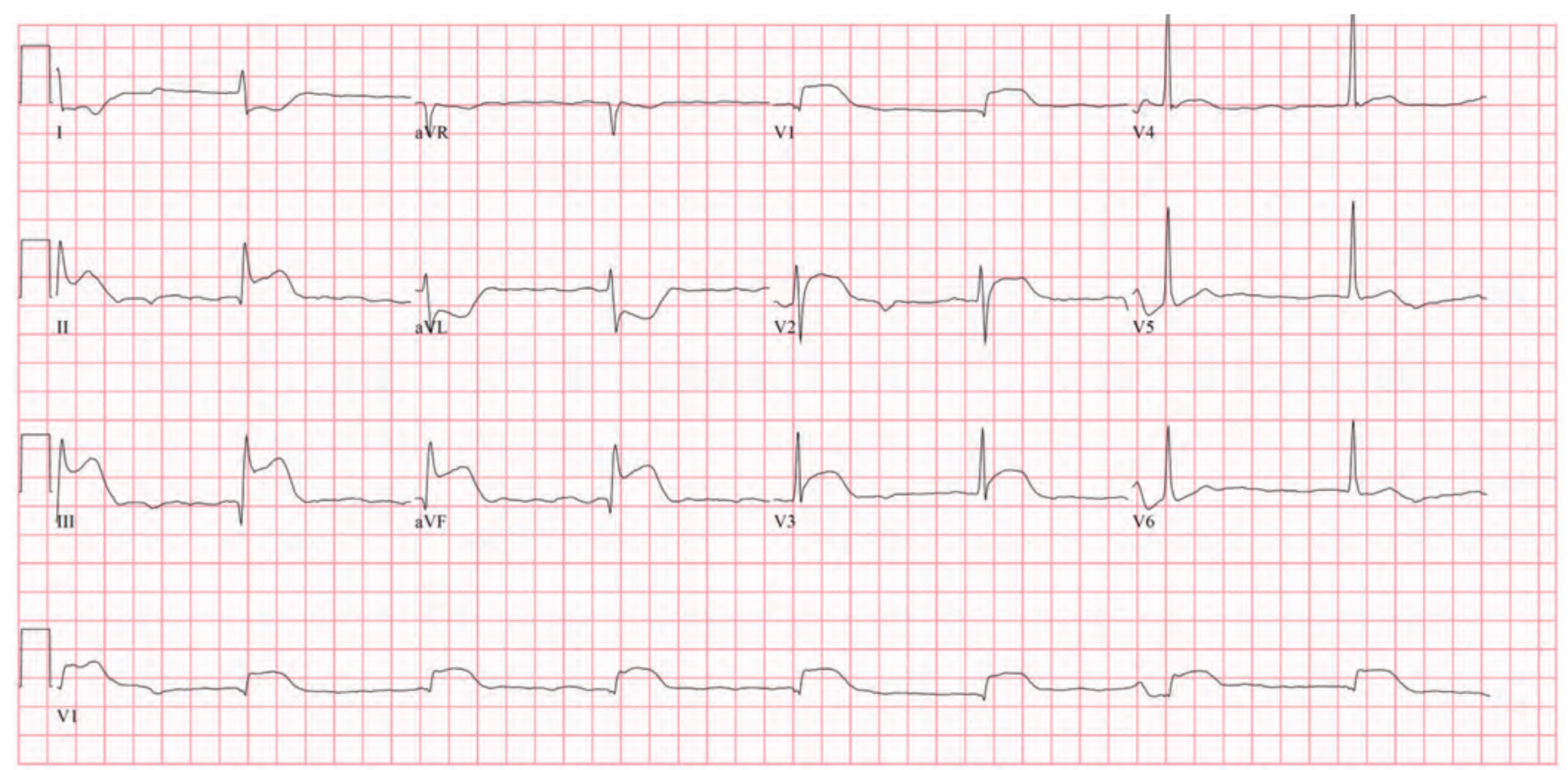

Figure 1: Electrocardiogram in a 55-year-old man showing junctional rhythm, right axis deviation and acute inferior and anterior ST-segment elevation myocardial infarction. Ventricular rate 47 beats/min, QRS duration 88 ms, QT/QTc 454/401. 
body temperature was cooled based on the recommended therapeutic hypothermia guidelines at the time, ${ }^{1,2}$ for targeted temperature between $32^{\circ} \mathrm{C}$ and $34^{\circ} \mathrm{C}$. His core temperature was continuously measured with a rectal thermistor attached to the cooling blanket. As his body temperature reached $32.4^{\circ} \mathrm{C}$, an ECG showed paroxysmal atrial fibrillation with pronounced Osborn waves and, most concerningly, a prolonged QTc at 654 ms (Figure 3); his electrolytes were normal. The patient began to have increased ventricular ectopy in left bundle branch block morphology precipitating ventricular fibrillation storm (Figure 4).

The patient received prompt and typical advanced cardiovascular life support, including intravenous anti-arrhythmic medication and multiple doses of magnesium sulfate. Active rewarming was started immediately by changing the cooling blanket to the

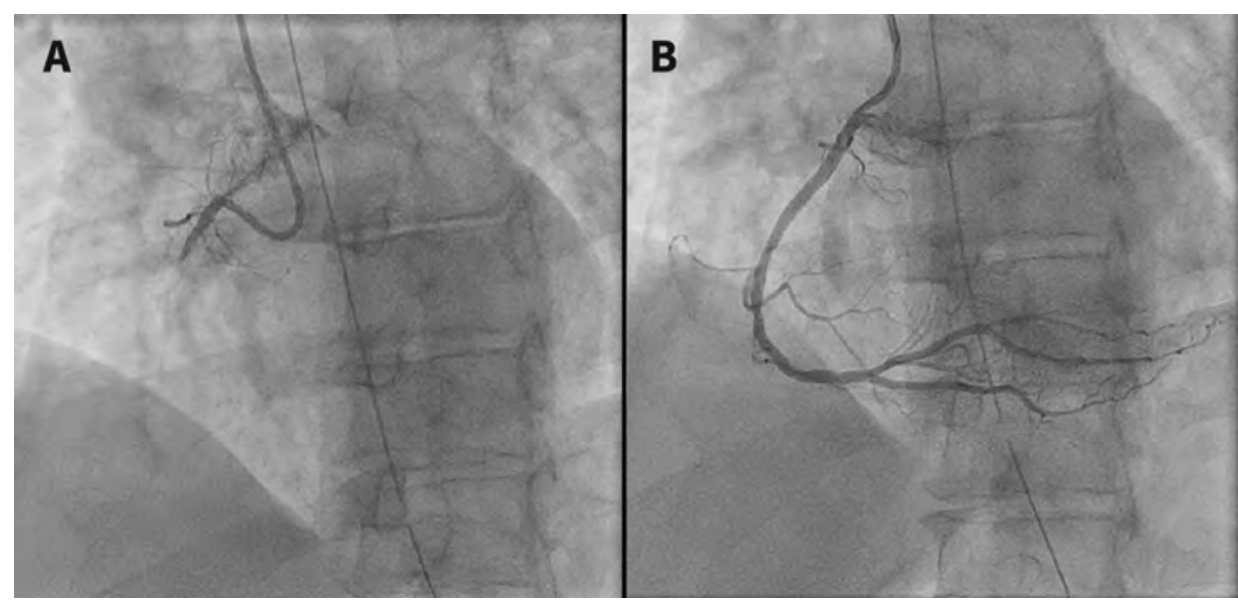

Figure 2: Two serial images of selective coronary angiograms showing the (A) proximally occluded right coronary artery (RCA) and (B) the subsequently revascularized RCA after percutaneous coronary intervention with one drug-eluting stent. active rewarming setting and applying a forced air-warming system to the patient's body. There were a total of 36 electrical defibrillations over about one hour, associated with intermittent CPR and resuscitation. Within 40 minutes of the onset of recurrent ventricular fibrillation, the patient was rushed back to the cardiac catheterization laboratory; the right coronary artery stent was patent. In case there was any ischemic precipitant to the arrest, at this point a drug-eluting stent was inserted into the critical second obtuse marginal artery with no resolution of the Osborn waves or prolonged QTC (Appendix 1, supplemental Figure 2). The patient continued to have episodes of ventricular fibrillation (Appendix 1, supplemental Figure 3). As the patient's body rewarmed and his body temperature reached $33.4^{\circ} \mathrm{C}$, another ECG showed prompt resolution of the Osborn waves and QTc shortened to $438 \mathrm{~ms}$ (Figure 5); the patient experienced no further ventricular fibrillation.

The patient successfully recovered and was discharged from the hospital seven days later with no neurologic sequelae from the prolonged arrest. He was assessed as an outpatient for possible channelopathies, including Brugada and long QT syndrome, by heart rhythm specialists, who felt these diagnoses were unlikely, given that all test results were normal and he had no family history of these conditions. His episodes of ventricular fibrillation were thought to have resulted from low core body temperature secondary to the therapeutic hypothermia protocol.

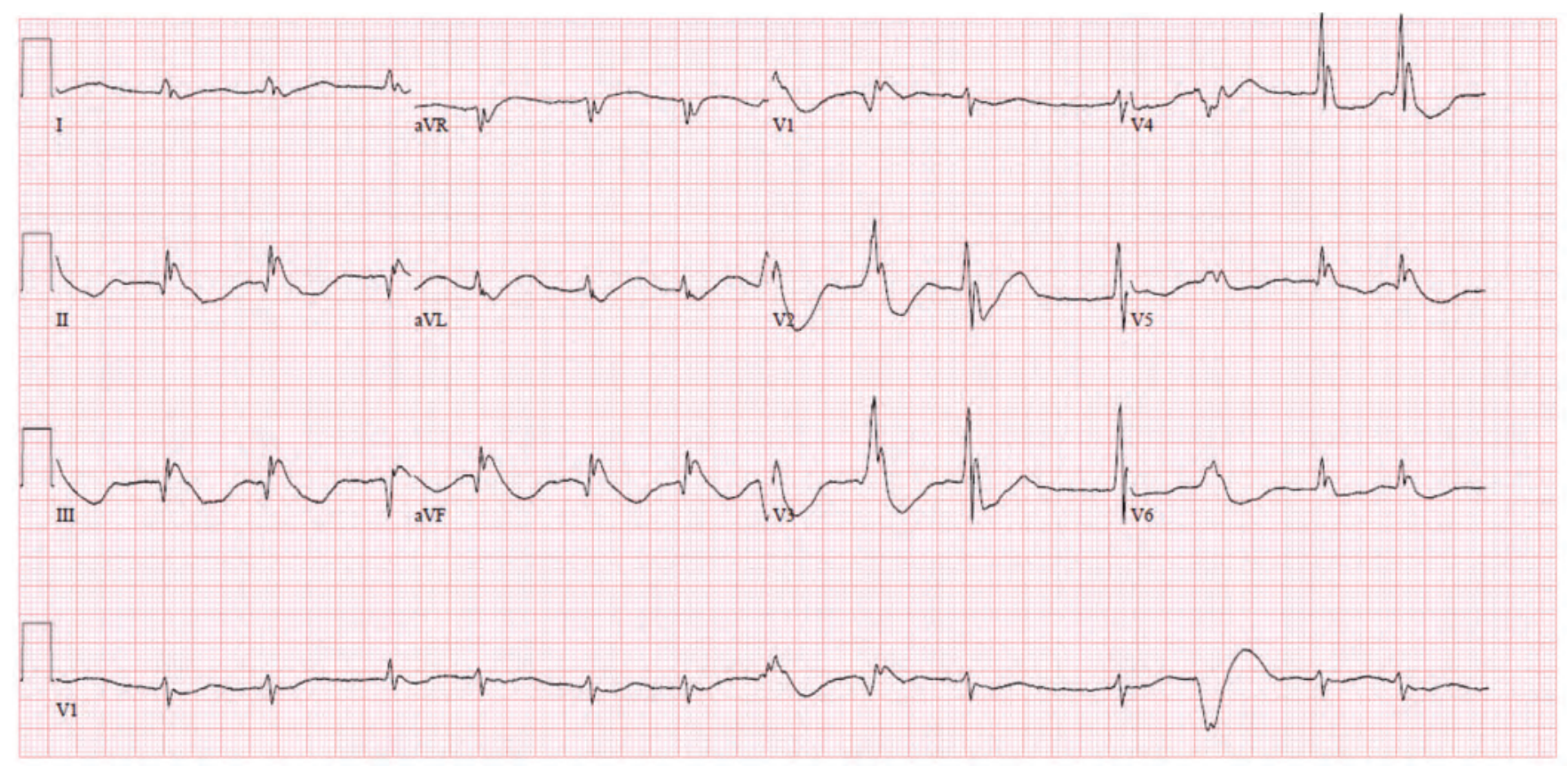

Figure 3: Electrocardiogram showing atrial fibrillation and Osborn waves. Extremely prolonged QTc with multifocal premature ventricular contractions. Ventricular rate 84 beats/min, QRS duration 146, QT/QTc 528/623. 


\section{Discussion}

For the last 15 years, optimal care for patients after cardiac arrest has included therapeutic hypothermia (also known as targeted temperature management), with goal core body temperature targets between $32^{\circ} \mathrm{C}$ and $34^{\circ} \mathrm{C}$, in an attempt to improve neurologic outcomes and mortality. ${ }^{1,2}$ Pathophysiological studies have shown that hypothermia decreases cerebral blood flow to the head, subsequently decreasing oxygen consumption by $7 \%$ to
$8 \%$ per $1^{\circ} \mathrm{C}$ reduction in core body temperature. ${ }^{3}$ This results in decreased cerebral metabolism and is thought to protect the brain from worsening anoxic injury. In 2002, two landmark randomized controlled trials (RCTs) provided evidence to support the basic science and theories behind therapeutic hypothermia after cardiac arrest. These two RCTs $\left(n=77^{1} ; n=275^{2}\right)$ involved participants who had remained unconscious after resuscitation from cardiac arrest, with an initial shockable rhythm. The trials compared therapeutic hypothermia (targeted temperature

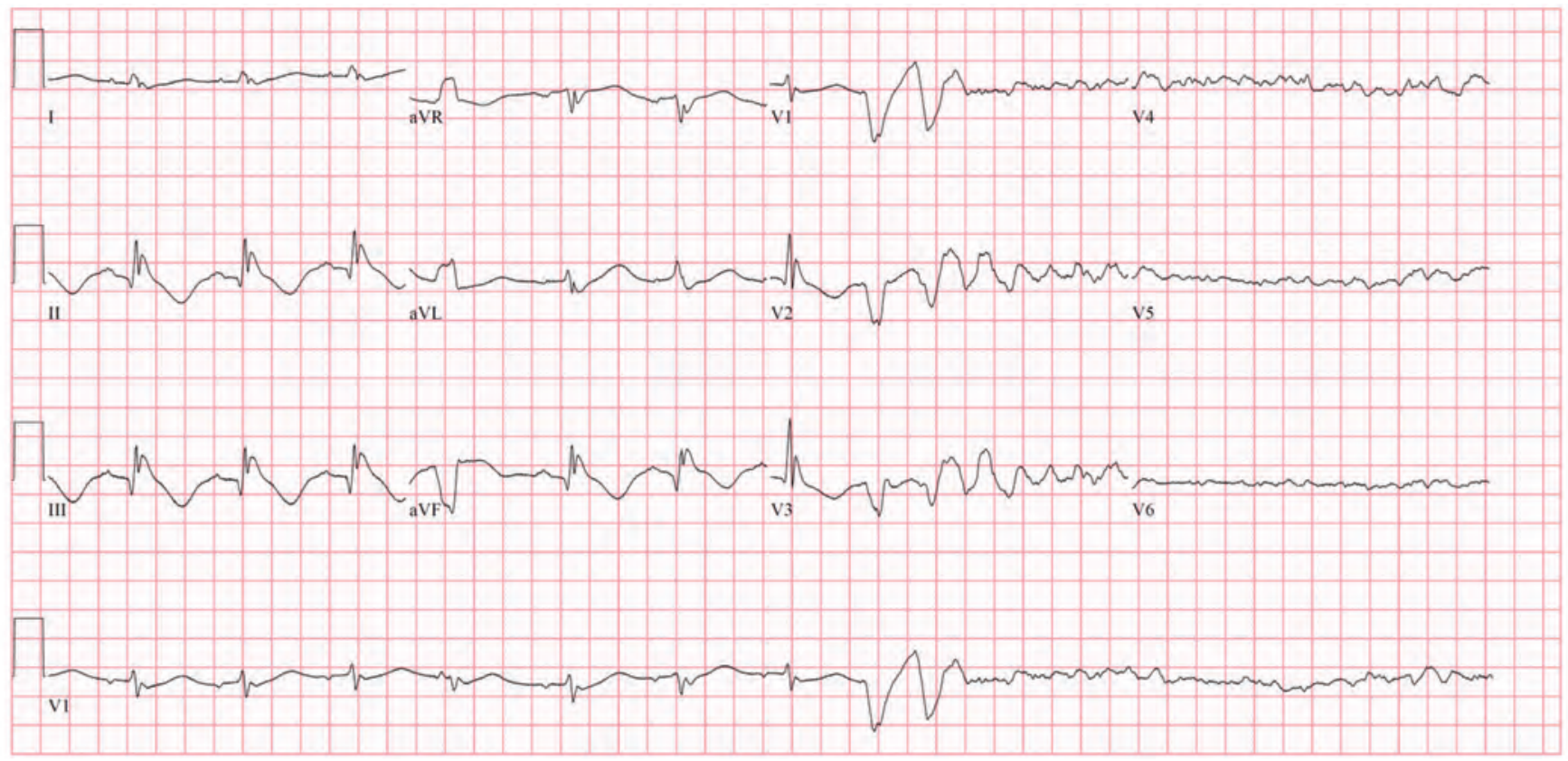

Figure 4: Electrocardiogram showing sinus rhythm with occasional premature ventricular contractions and extremely prolonged QTc, followed by ventricular fibrillation. Initial ventricular rate 104 beats/min, QRS duration 158 ms, QT/QTC 498/654.

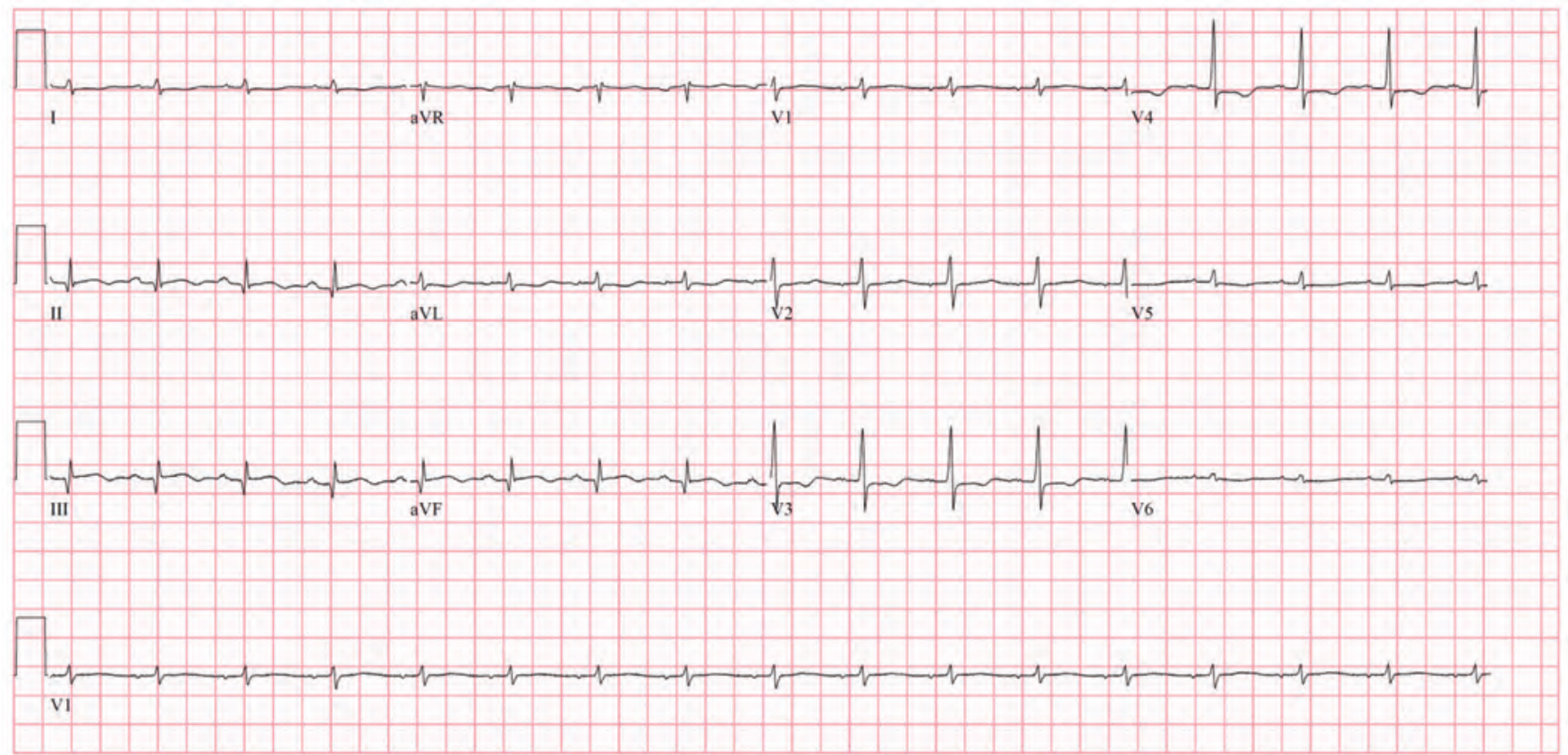

Figure 5: Electrocardiogram showing normal sinus rhythm. Serial changes of acute inferior infarct, with drastic decrease in the previously prolonged QTc are visible. Ventricular rate 98 beats/min, PR interval 156 beats/min, QRS duration 74 ms, QT/QTc 392/500. 
between $32^{\circ} \mathrm{C}$ and $34^{\circ} \mathrm{C}$ for 12 to 24 hours) with standard treatment after cardiac arrest. Both studies showed statistically and clinically significant increases in the rate of favourable neurologic outcomes in the hypothermia group.,2 The second study also showed reduced mortality (risk ratio $0.74,95 \%$ confidence interval $0.58-0.95, p=0.02$ ) in the therapeutic hypothermia group) compared with participants who were normothermic. ${ }^{2}$ These two studies resulted in broad changes to care after cardiac arrest, and the beginning of use of therapeutic hypothermia protocols after arrest around the world.

Recently, the benefit of therapeutic hypothermia protocols in patients after cardiac arrest has been questioned. Criticisms of the two previously mentioned RCTs included the small sample sizes. In addition, in one of the RCTs, fever developed in many of the patients in the normothermic group (average temperature $\left.37.8^{\circ} \mathrm{C}\right) .^{2}$ Fever or hyperthermia in a patient after cardiac arrest is associated with adverse neurologic outcomes. ${ }^{4}$ That RCT also excluded $90 \%$ of participants who were randomly assigned, which raises the question of the broad generalizability of its findings.

Given these uncertainties, new investigations were conducted. An international multicentre RCT that compared two targeted temperature regimens was completed in 2013. ${ }^{4}$ The study ${ }^{4}$ involved 939 unconscious adult participants who survived outof-hospital cardiac arrest of presumed cardiac cause and compared those with mild hypothermia (targeted temperature of $33^{\circ} \mathrm{C}$ ) versus those with a targeted, controlled body temperature $\left(36^{\circ} \mathrm{C}\right) .^{2,4}$ There was no significant difference between the two groups in neurologic outcome and mortality. Some researchers have interpreted the study as support for an optimal targeted temperature between $33^{\circ} \mathrm{C}$ and $36^{\circ} \mathrm{C}$, but others have suggested that simply preventing hyperthermia and fevers may suffice. ${ }^{2,4}$

\section{Adverse cardiac effects of hypothermia}

Although there are no documented cases of fatal arrhythmias secondary to effects of the therapeutic hypothermia protocol, several prospective studies have reported minor arrhythmias and QTC prolongation..$^{5-7}$ In one of these studies, new arrhythmias occurred in $38.3 \%$ of patients. However, none of these patients had sustained ventricular tachycardia, polymorphic ventricular tachycardia or ventricular fibrillation. The noted arrhythmias were nonsustained ventricular tachycardia (19.1\%), sinus bradycardia (10.6\%), accelerated nodal rhythm (8.5\%) and atrial fibrillation (6.4\%). ${ }^{5}$ These three studies evaluated the effect of therapeutic hypothermia on QTc length and showed a statistically significant increase in the mean baseline QTc, which initially ranged from 400 to $440 \mathrm{~ms}$ before cooling to a mean of 510 to 550 ms when core body temper- ature was $32^{\circ} \mathrm{C}-34^{\circ} \mathrm{C} . .^{5-7}$ However, despite the increased QTc and QTc prolongation above $600 \mathrm{~ms}$ in some participants, none of the participants in the three prospective studies had fatal arrhythmias. There are two case reports in the literature regarding therapeutic hypothermia after cardiac arrest in patients with documented long QT syndrome, in which the authors postulate that cooling is beneficial and safe in this population..$^{8,9}$

\section{Current recommendations}

The Canadian Cardiovascular Society, Canadian Cardiovascular Critical Care Society and Canadian Association of Interventional Cardiology released new recommendations in 2017 that support a targeted body temperature range between $33^{\circ} \mathrm{C}$ and $36^{\circ} \mathrm{C}$ for patients who undergo targeted temperature management. ${ }^{10}$

\section{Conclusion}

Although generally considered a safe and beneficial procedure, therapeutic hypothermia can prolong QTc intervals and may uncommonly result in life-threatening arrhythmias, as described in our case. Current protocols in hospitals should be reviewed to consider revised recommendations for body temperature targets, which may reduce the risk of serious hypothermia-related arrhythmias.

\section{References}

1. Bernard SA, Gray TW, Buist MD, et al. Treatment of comatose survivors of out-of hospital cardiac arrest with induced hypothermia. N Engl J Med 2002;346:557-63.

2. Hypothermia after Cardiac Arrest Study Group. Mild therapeutic hypothermia to improve the neurologic outcome after cardiac arrest. N Engl J Med 2002;346:549-56.

3. Wyllie J, Perlman JM, Kattwinkel J, et al. Neonatal Resuscitation Chapter Collaborators. Part 11: Neonatal resuscitation: 2010 International Consensus on Cardiopulmonary Resuscitation and Emergency Cardiovascular Care Science with Treatment Recommendations. Resuscitation 2010;81(Suppl 1):e260-87.

4. Nielsen N, Wetterslev J, Cronberg T, et al.; TTM Trial Investigators. Targeted temperature management at $33^{\circ} \mathrm{C}$ versus $36^{\circ} \mathrm{C}$ after cardiac arrest. N Engl J Med 2013; 369:2197-206.

5. Salinas P, Lopez-de-Sa E, Pena-Conde L, et al. Electrocardiographic changes during induced therapeutic hypothermia in comatose survivors after cardiac arrest. World J Cardiol 2015;7:423-30.

6. Storm C, Hasper D, Nee J, et al. Severe QTc prolongation under mild hypothermia treatment and incidence of arrhythmias after cardiac arrest - a prospective study in 34 survivors with continuous Holter ECG. Resuscitation 2011;82:859-62.

7. Weitz D, Greet B, Bernstein SA, et al. The benign nature of mild induced therapeutic hypothermia - induced long QTc. Int J Cardiol 2013;168:1583-5.

8. Jatti K, Prasad N. Therapeutic hypothermia after cardiac arrest in long QT syndrome: Could it be an adjunctive treatment to prevent dysrhythmias? SAGE Open Med Case Rep 2015;3:2050313X15581267.

9. Nishiyama N, Sato T, Aizawa Y, et al. Extreme QT prolongation during therapeutic hypothermia after cardiac arrest due to long QT syndrome. Am J Emerg Med 2012;30:638.e5-8.

10. Wong GC, van Diepen S, Ainsworth C, et al.; CCS Post Cardiac Arrest Guidelines Committee. Canadian Cardiovascular Society/Canadian Cardiovascular Critical Care Society/Canadian Association of Interventional Cardiology position statement on the optimal care of the postarrest patient. Can J Cardiol 2017;33:1-16.

\section{Competing interests: None declared.}

This article has been peer reviewed.

The authors have obtained patient consent.

Affiliations: Department of Medicine (Moeller, Webber), Dalhousie University; Queen Elizabeth II Health Science Centre (Moeller, Webber), Halifax, NS
Contributors: Andrew Moeller and Jordan Webber submitted this manuscript for publication. Both authors contributed to the conception and design of the work, drafted the manuscript, revised it critically for important intellectual content, gave final approval of the version to be published and agreed to be accountable for all aspects of the work.

Correspondence to: Andrew Moeller, andrewmoeller@gmail.com 